\title{
Presencia del Apéndice Fibroso Hepático: Estudio de Correlaciones Bioantropológicas y su Estructura
}

\author{
Presence of Appendix Fibrosa Hepatitis: Study of Bioanthropological and Structure Correlation \\ Oñate, J. C.; Sanhueza, P.; Alarcón, E.; López, R.; Montero, C. \& Mandiola, E.
}

OÑATE, J. C.; SANHUEZA, P.; ALARCóN, E.; LÓPEZ, R.; MONTERO, C. \& MANDIOLA, E. Presencia del apéndice fibroso hepático: estudio de correlaciones bioantropológicas y su estructura. Int. J. Morphol., 23(3):217-219, 2005.

RESUMEN: El apéndice fibroso hepático es una estructura anatómica del espacio subfrénico, poco descrita en la literatura universal y de importancia clínica. Se diseñó un protocolo para ser realizado en cadáveres donde se determina su presencia, características y correlación estadística con factores antropométricos. En 24 cadáveres se determina que el apéndice fibroso hepático siempre está presente, y que no existe correlación estadísticamente significativa, pero sí se observa variabilidad entre las dimensiones biométricas analizadas.

PALABRAS CLAVE : Hígado; Apéndice fibroso del hígado; Biometría.

\section{INTRODUCCIÓN}

Al revisar en la Anatomía clásica (Warwick \& Williams, 1979) los elementos de fijación del hígado, se describe la presencia del ligamento triangular izquierdo y una prolongación libre de éste, el cual se extiende desde el lado lateral del lóbulo izquierdo del hígado, en el adulto, hacia el diafragma, el cual es llamado apéndice fibroso y que representa los resquicios atrofiados del lóbulo izquierdo y puede contener restos de conductos bilíferos, conocidos como vasos aberrantes del hígado. En la Clínica actual, Gao et al. (1980) y Gao \& Roberts (1986), insisten en el cuidado y observación de esta estructura en una cirugía de hígado.

Las estructuras presentes en el apéndice fibroso pueden incluir, además de los conductos biliares aberrantes, vasos sanguíneos, células hepáticas y fibras nerviosas. Cabe destacar que se han descrito casos de peritonitis biliar post operatoria en pacientes operados de gastrectomía, como también variaciones de la vena frénica inferior izquierda presentes en el apéndice fibroso y complicaciones hemostáticas post operatorias cuando éstas están presentes. Estos dos últimos puntos, hacen que los actos quirúrgicos adquieran vital importancia cuando se accede a esta zona anatómica (Champetier et al., 1982; Bonnette \& Gayet, 1983 e Iso et al., 1996).
Motivados por la escasa la literatura al respecto, en el presente trabajo se describe el apéndice fibroso del hígado y sus dimensiones en especímenes adultos, realizando su estudio con relación a sexo, edad y factores biométricos.

\section{MATERIAL Y MÉTODO}

El apéndice fibroso del hígado fue estudiado en 24 cadáveres adultos, de ambos sexos, provenientes de autopsias, en los cuales se consignó sexo, edad, peso, talla, peso del hígado, y medidas en ancho (dimensión máxima entre los bordes del apéndice fibroso) y largo del apéndice fibroso (esto es, la distancia lineal a partir del extremo izquierdo del hígado y su inserción en el diafragma) (Fig. 1).

Se realizó análisis estadístico de las variables mediante la aplicación del software Graphpad Prism versión 3.2, utilizando el test de correlación de Pearson, t Student y ANOVA. 


\section{RESULTADOS}

En un total de 24 cadáveres, 6 correspondían al sexo femenino y 18 al masculino. La edad promedio fue de 43 años (rango de 16 a 75 años), la talla promedio fue de 165 $\mathrm{cm}$ (rango de 140 a $185 \mathrm{~cm}$ ) y el peso promedio fue de 66,2 $\mathrm{kg}$ (rango de 26,3 a $87,5 \mathrm{~kg}$ ).

Se observa que el peso promedio del hígado fue de 1.535 g (rango de 570 a 3.020 g). En los cadáveres estudiados, el apéndice fibroso hepático estaba presente en el $100 \%$ de los casos, y sus medidas en promedio eran $22 \mathrm{~mm}$ de ancho (rango de 5 a $60 \mathrm{~mm}$ ) y de $66 \mathrm{~mm}$ de largo (rango de 35 a $125 \mathrm{~mm}$ ). (Fig. 1).

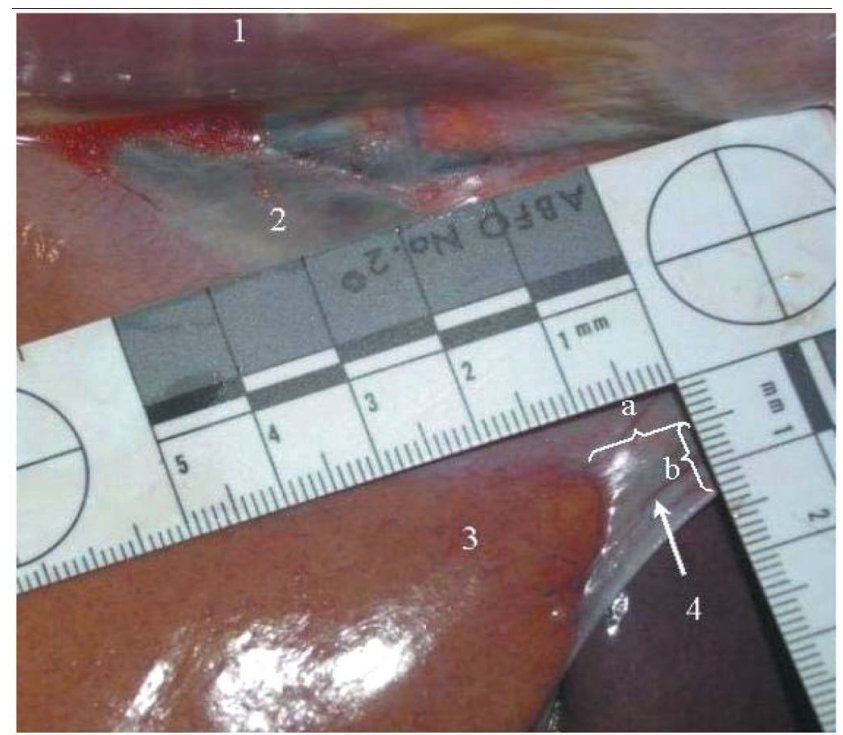

Fig. 1. Vista anterior del hígado. 1. Diafragma; 2. Ligamento triangular izquierdo; 3. Lóbulo izquierdo; 4. Apéndice fibroso del hígado; a. Largo; b. Ancho.

Al analizar los resultados estadísticos, se puede observar que no existe correlación $(\mathrm{p}=0,749)$ entre el largo del apéndice fibroso y el peso del hígado (Fig. 2). Al analizar las variables ancho del apéndice fibroso hepático y peso hepático, si bien no existe una correlación estadísticamente significativa ( $\mathrm{p}=0,149)$, es posible apreciar una leve tendencia a que un mayor peso hepático se relaciona con un mayor ancho del apéndice fibroso (Fig. 3).

Para las otras variables analizadas, ancho del apéndice v/s largo del apéndice, y área del apéndice fibroso v/s peso hepático, no existe una correlación estadísticamente significativa, con valores de p de 0,3327 y 0,2567 , respectivamente (Figs. 4 y 5 ).

La Fig. 6 muestra la presencia de vasos sanguíneos en el espesor del apéndice fibroso.

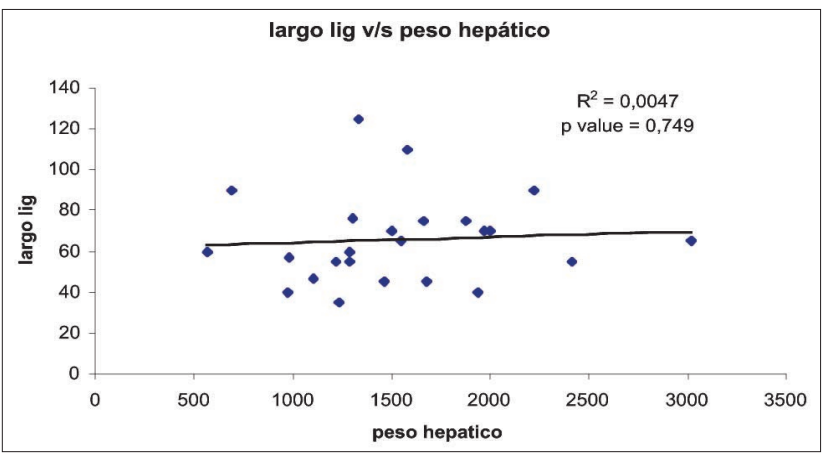

Fig. 2. Correlación estadística entre el largo del apéndice versus peso hepático.

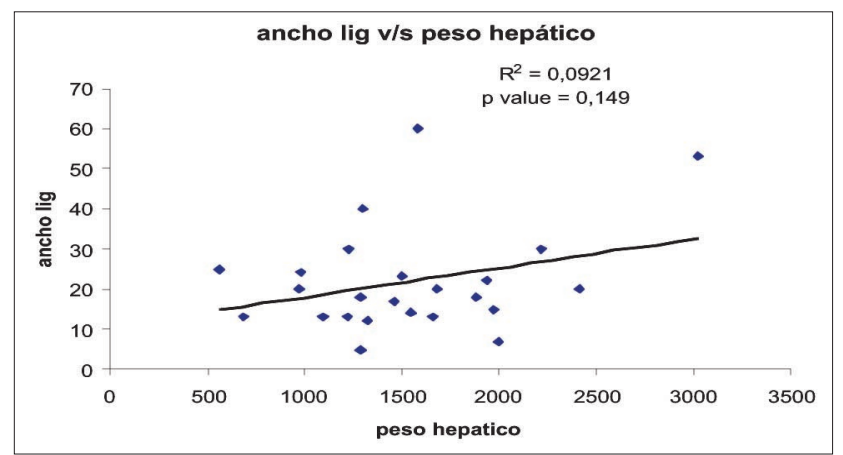

Fig. 3. Correlación estadística entre el ancho del apéndice versus peso hepático.

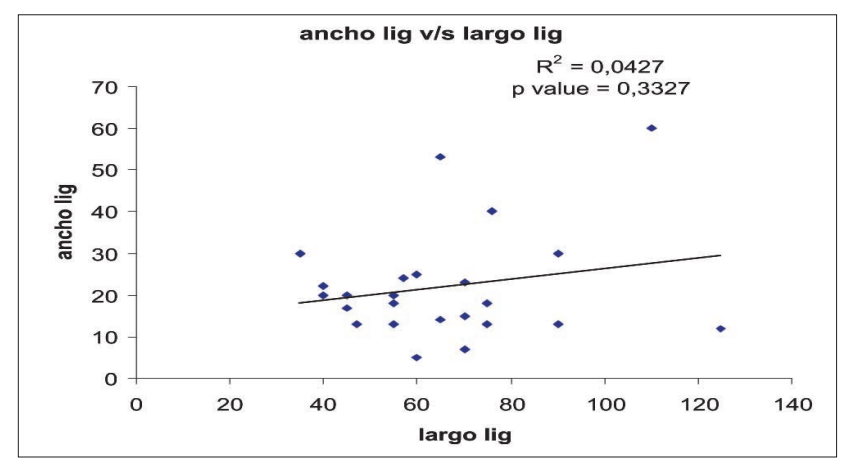

Fig. 4. Correlación estadística entre el ancho del apéndice versus el largo del apéndice.

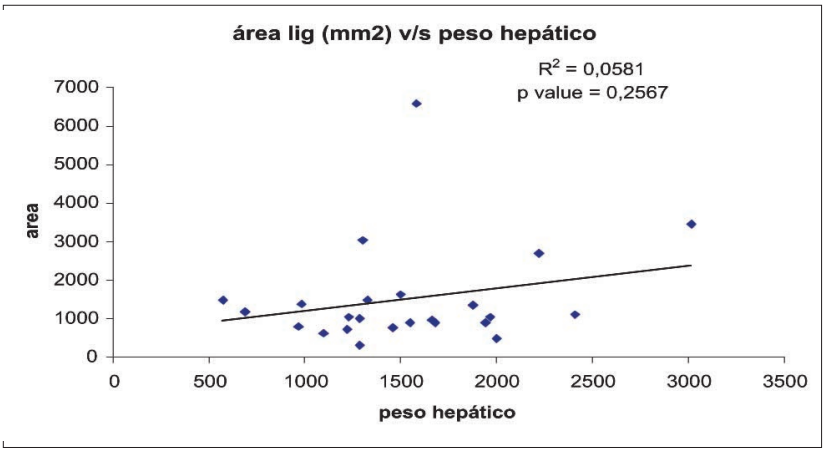

Fig. 5. Correlación estadística área del apéndice versus el peso hepático. 


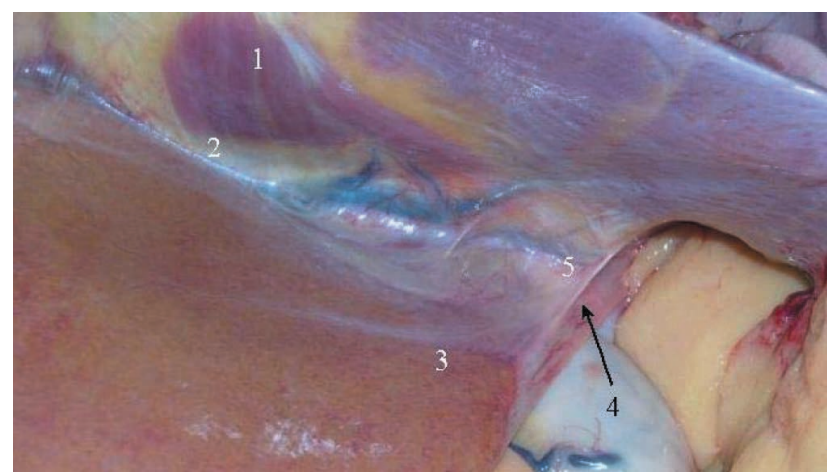

Fig. 6. Vista anterior del hígado. 1. Diafragma; 2. Ligamento triangular izquierdo; 3. Lóbulo izquierdo; 4. Apéndice fibroso del hígado; 5 . Vasos sanguíneos en el espesor del apéndice.

\section{DISCUSIÓN}

La revisión de la literatura, demuestra que es reducida la descripción referida al apéndice fibroso del hígado. Los anatomistas clásicos no son precisos en su descripción . Warwick \& Williams lo refieren más bien como vestigio embrionario, incluso cuando en su espesor contiene vasos sanguíneos y ductos bilíferos aberrantes y Feneis \& Dauber lo describen como una estructura no constante, sin aportar mayores detalles. Sin embargo, el apéndice fibroso del hígado lo encontramos en el $100 \%$ de los casos.

Cabe destacar que en la revisión de la bibliografía actual, especialmente clínica, corresponde a una entidad importante, en la que se han descrito elementos vasculares y biliares, los que deberían ser considerados al momento de realizar accesos quirúrgicos a la región, con el fin de evitar complicaciones en el post operatorio; esto último demostrado en los estudios de Bonette \& Gayet; Champetier et al. e Iso et al.

En los especímenes estudiados, se pudo establecer que las medidas promedio fueron de $2,2 \mathrm{~cm}$ de ancho $6,6 \mathrm{~cm}$ de largo. Pese a lo variable de las dimensiones, no observamos una correlación estadísticamente significativa entre los parámetros considerados.

OÑATE, J.C.; SANHUEZA, P.; ALARCÓN, E.; LÓPEZ, R.; MONTERO, C. \& MANDIOLA, E. Presence of appendix fibrosa hepatis : study of bioanthropological and structure correlation Int. J. Morphol., 23(3):217-219, 2005.

SUMMARY: The hepatic fibrous appendix is an anatomical structure of the subfrenic space scarcely described in the universal literature and of vital clinical importance. For their study a protocol was designed to be carried out in cadavers where its presence is determined, characteristic and statistical correlation with anthropometric factors. In 24 cadavers it is determined that the hepatic fibrous appendix is always present, and that correlation doesn't exist statistically significant among the analyzed anthropological factors, but it's possible to observe variability in it's dimensions.

KEY WORDS : Liver; Appendix fibrosa hepatis; Biometry.

\section{REFERENCIAS BIBLIOGRÁFICAS}

Bonnette, P. \& Gayet, B. Section du ligament triangulaire gauche du foie. Rapports des veines diaphragmatique et sushépatique gauches. Presse Médicale, 12(10):639-40, 1983.

Champetier, J.; Davin, J. L.; Letoublon, C. et al. Aberrant biliary ducts (vasa aberrantia): Surgical implications. Anat. Clin., 4:137-45, 1982.

Gao, X. H. \& Roberts, A. The left triangular ligament of the liver and the structures in its free edge (appendix fibrosa hepatis) in chinese and canadian cadavers. Am. Surgeon, 52:246-52, 1986 .

Gao, X. H.; Lei, Q. F.; Zhou, D. M. \& Min, P. Q. The study of the positions of the coronary ligaments and the left triangular ligament of the liver in relation to the subdivision of the subphrenic space. Acta Academiae Medicinae Sicuani, 11:107-15, 1980 .
Iso, Y.; Kusaba, I.; Matsumata, T. et al. Postoperative bile peritonitis caused by division of an aberrant bile duct in the left triangular ligament of the liver. Am. J. Gastroenterol., 91(11):2428-30, 1996.

Warwick, R. \& Williams, P. Gray Anatomia. 35 ed., Guanabara Koogan, Rio de Janeiro, 1979. V. II.

Dirección para correspondencia:

Dr. Iuan Carlos Oñate Facultad de Ciencias de la Salud

Depto. de Ciencias Biológicas. Área de Anatomía.

Universidad Andrés Bello

Avda República 217.

Santiago, CHILE. 
